\title{
Comparative assessment of An. gambiae and An. stephensi mosquitoes to determine transmission-reducing activity of antibodies against $P$. falciparum sexual stage antigens
}

Maarten Eldering ${ }^{1 \dagger}$, Anaïs Bompard ${ }^{2 \dagger}$, Kazutoyo Miura ${ }^{3}$, Will Stone ${ }^{1}$, Isabelle Morlais ${ }^{4}$, Anna Cohuet ${ }^{4}$, Geert-Jan van Gemert ${ }^{1}$, Patrick M. Brock ${ }^{2,6}$, Sanna R. Rijpma ${ }^{1}$, Marga van de Vegte-Bolmer ${ }^{1}$, Wouter Graumans ${ }^{1}$, Rianne Siebelink-Stoter ${ }^{1}$, Dari F. Da ${ }^{5}$, Carole A. Long ${ }^{3}$, Merribeth J. Morin ${ }^{7}$, Robert W. Sauerwein ${ }^{1}$, Thomas S. Churcher ${ }^{2}$ and Teun Bousema ${ }^{1,8^{*}}$

\begin{abstract}
Background: With the increasing interest in vaccines to interrupt malaria transmission, there is a demand for harmonization of current methods to assess Plasmodium transmission in laboratory settings. Potential vaccine candidates are currently tested in the standard membrane feeding assay (SMFA) that commonly relies on Anopheles stephensi mosquitoes. Other mosquito species including Anopheles gambiae are the dominant malaria vectors for Plasmodium falciparum in sub-Saharan Africa.

Methods: Using human serum and monoclonal pre-fertilization (anti-Pfs48/45) and post-fertilization (anti-Pfs25) antibodies known to effectively inhibit sporogony, we directly compared SMFA based estimates of transmissionreducing activity (TRA) for An. stephensi and An. gambiae mosquitoes.

Results: In the absence of transmission-reducing antibodies, average numbers of oocysts were similar between An. gambiae and An. stephensi. Antibody-mediated TRA was strongly correlated between both mosquito species, and absolute TRA estimates for pre-fertilisation monoclonal antibodies (mAb) showed no significant difference between the two species. TRA estimates for lgG of naturally exposed individuals and partially effective concentrations of anti-Pfs $25 \mathrm{mAb}$ were higher for An. stephensi than for An. gambiae.

Conclusion: Our findings support the use of An. stephensi in the SMFA for target prioritization. As a vaccine moves through product development, better estimates of TRA and transmission-blocking activity (TBA) may need to be obtained in epidemiologically relevant parasite-species combination.
\end{abstract}

Keywords: Malaria, Anopheles, Transmission, Vaccine, Immunity

\footnotetext{
* Correspondence: teun.bousema@radboudumc.nl

${ }^{\dagger}$ Equal contributors

'Department of Medical Microbiology, Radboud University Medical Center,

Nijmegen, The Netherlands

${ }^{8}$ Department of Immunology and Infection, London School of Hygiene and

Tropical Medicine, London, UK

Full list of author information is available at the end of the article
} 


\section{Background}

Recent declines in Plasmodium falciparum malaria transmission intensity in several African settings, associated with the wide-scale deployment of efficacious vector control and artemisinin combination therapy [1], have contributed to a renewed interest in the elimination of malaria. Further scaling up of control efforts with currently available tools is unlikely to achieve this goal in most African settings [2] and interventions that specifically target malaria transmission are considered highly desirable to accelerate elimination efforts [3]. Vaccines that interrupt malaria transmission (VIMT) are high on the priority list for malaria elimination [4]. These include classical transmission-blocking vaccines that target sexual, sporogonic, or mosquito stages of the parasite (SSM-VIMT) and interfere with parasite transmission in the vector host. Recent success in obtaining properly folded vaccine candidates that elicit functional transmission-blocking immunity in animal models have paved the way for clinical development of lead SSM-VIMT candidates (reviewed in [5-7]). For P. falciparum, these candidates include vaccines based on parasite antigens Pfs230, Pfs48/45, Pfs25 and mosquito antigen AnAPN1 [5] acting on different life-stages during sporogonic development.

Malaria transmission starts with the ingestion of male and female gametocytes by blood-feeding anophelines. Upon ingestion, gametocytes quickly differentiate into male microgametes and female macrogametes that fuse to form zygotes. Within $18-24 \mathrm{~h}$ this zygote turns into a motile ookinete which penetrates the mosquito midgut epithelium and differentiates into an oocyst [8]. Over the next two weeks sporozoites do develop inside the oocyst, eventually bursting the oocyst capsule before migrating to and invading the salivary glands, rendering the mosquito infective to humans. Both vaccine-induced and naturally acquired antibodies against Pfs 230 and Pfs48/ 45 can prevent fertilization and thus zygote formation [9-12]. Antibodies against Pfs25 prevent ookinete penetration of the mosquito midgut $[13,14]$ while antibodies against AnAPN1 also block ookinete penetration by preventing the parasite interacting with mosquito midgut ligands $[15,16]$.

Clinical development of SSM-VIMTs has been primarily guided by assessments of TRA in the SMFA that utilizes the African $P$. falciparum strain NF54 and Anopheles stephensi mosquitoes [17, 18]. The SMFA also forms the gold standard assay to assess naturally acquired transmission-reducing immunity [18]. Anopheles stephensi is a primary malaria vector in the Indian subcontinent but not in Africa where A. funestus and An. gambiae (s.l.) dominate [19]. Anopheles stephensi and An. gambiae mosquitoes belong to the subgenus Cellia, a group of major Old World malaria vectors with a wide geographical distribution that diversified millions of years ago [20]. There are several factors in mosquito physiology and immunity that may contribute to differences in the vectorial competence of An. stephensi and An. gambiae [21-24]. These potential differences raise questions about the extent to which SMFA results, commonly using An. stephensi, can be directly translated to An. gambiae. Here, we compared An. stephensi and An. gambiae in terms of transmission efficiency and TRA estimates in the SMFA using transmission-blocking mAb and sera of naturally gametocyte-exposed individuals.

\section{Methods \\ Mosquito rearing, parasite culture and standard membrane feeding assay}

Anopheles stephensi (Sind-Kasur Nijmegen strain) [25] and An. gambiae (s.s.) (Ngousso strain) [26] were reared at $30{ }^{\circ} \mathrm{C}$ and $70-80 \%$ humidity, and exposed to a $12 /$ $12 \mathrm{~h}$ day/night cycle. Mature P. falciparum (NF54) gametocytes (14 day culture, $0.3-0.5 \%$ gametocytes, $2 \%$ haematocrit) were obtained from an automated tipper system and prepared as previously described [27, 28]. For infection experiments, 3-5 day-old mosquitoes were fed on a glass membrane feeder system containing $270 \mu \mathrm{l}$ of P. falciparum culture mixture [28]. Unfed and partially fed mosquitoes were removed from the samples. Blood-fed mosquitoes were maintained at $26{ }^{\circ} \mathrm{C}$ and $70-80 \%$ humidity. Routine dissection and staining of midguts was done 6-9 days post-infection in $1 \%$ mercurochrome solution for oocysts quantification.

\section{Antibody preparations Monoclonal antibodies}

Four monoclonal antibodies against two $P$. falciparum antigens were selected for the SMFA species comparison. Rat mAb 85RF45.1 and 85RF45.5, against prefertilization protein Pfs48/45 epitopes 1 and 5, respectively [29] and rat mAb 32F81 [30] and mouse mAb 4B7 against [31] post-fertilization protein Pfs25. Concentrations of $\mathrm{mAb}$ were chosen to achieve full and partial TRA in An. stephensi. All mAb were diluted in human serum aiming for a final concentration in the feeder for Pfs48/45-85RF45.1: 10, 2.5, 0.63 and $0.16 \mu \mathrm{g} / \mathrm{ml}$; Pfs48/ 45-85RF45.5: 60, 20, 6.7 and $2.2 \mu \mathrm{g} / \mathrm{ml}$; Pfs25-4B7: 94, 23.5, 5.9 and $1.47 \mu \mathrm{g} / \mathrm{ml}$; Pfs25-32F81: 15, 10, 5, 2.5, $1.67,1.25,0.63$ and $0.56 \mu \mathrm{g} / \mathrm{ml}$. Human serum was used as a control for TRA assessments. In the models that assessed the association between antibody concentration and TRA, we also included other experiments conducted with the same mAb in the same lab in the period 19982015 with additional antibody concentrations for Pfs48/ 45-85RF45.1 (20-0.04 $\mu \mathrm{g} / \mathrm{ml})$, Pfs48/45-85RF45.5 (100$6.25 \mu \mathrm{g} / \mathrm{ml})$ and Pfs25-32F81 $(20-0.63 \mu \mathrm{g} / \mathrm{ml})$. 


\section{Human test sera}

Two sets of plasma samples were selected for analysis in the SMFA species comparison. One set $(n=5)$ comprised plasma samples from European expatriates who lived in malaria endemic regions for more than 10 years, and showed strong and consistent TRA in the SMFA [18, 32]. A second set $(n=21)$ comprised plasma samples derived from gametocyte carriers living in endemic regions in Cameroon (Yaoundé, 2010-2012) and Burkina Faso (Bobo-Dioulasso, 2012). Samples were collected for ex vivo infectivity assessments as reported previously [33]. Ethical approval was obtained from the Centre Muraz Institutional Ethics Committee under agreement number 0003-2009/ CE-CM. The protocol conforms to the declaration of Helsinki on ethical principles for medical research involving human subjects (version 2002) and informed written consent were obtained from all volunteers.

IgG was purified from $300 \mu \mathrm{l}$ plasma samples using Protein G HP Spintrap (GE Healthcare, Chicago, IL, US) according to the manufacturer's instructions and as previously described $[18,34]$ and reconstituted in $300 \mu \mathrm{l}$ of milliQ water. Yield was $70-80 \%$ and IgG concentrations were $7-18 \mathrm{mg} / \mathrm{ml}$ measured with the NanoDrop ND1000 (Thermo Scientific, Waltham, MD, US). IgG was tested by mixing $90 \mu \mathrm{l}$ of plasma IgG in freeze-dried foetal calf serum (FCS) (original volume was $90 \mu \mathrm{l}$ ) and added to $180 \mu \mathrm{l}$ of gametocyte/red blood cell mix (containing $30 \mu \mathrm{l}$ of active complement containing serum) to a total volume of $270 \mu \mathrm{l}$. Primary control samples were $90 \mu \mathrm{l}$ of freeze-dried FCS dissolved in $90 \mu \mathrm{l}$ milliQ water. Subsidiary controls (IgG without TRA from an expatriate and individuals from Cameroon and Burkina Faso) were also tested. In addition, $30 \mu \mathrm{l}$ of active complement containing human serum (Sanquin Blood Supply, Nijmegen, Netherlands) was included in the culture mix for all serum IgG experiments. No significant differences were observed between IgG and FCS controls. Human IgG controls and FCS were used as the primary controls as they were available for all experiments. All SMFAs were blinded for evaluation and analysis.

\section{Statistical analysis}

For our analysis we only included experiments with a minimum of 20 dissected mosquitoes per group and a minimum infection prevalence of $70 \%$ in the control serum/IgG group (excluded experiments available in Additional file 1: Table S1) [32]. Statistical analyses were performed using $\mathrm{R}$ software (v 3.2 [35])) and $\mathrm{R}$ package $\operatorname{glmm} A D M B$ (v 11.6, [36]). TRA was estimated as the relative reduction in oocyst intensity in the treatment relative to the control. First, Spearman's correlation tests were performed on TRA results for both species to determine whether use of one species over another would influence candidate prioritization. Subsequently, generalized linear mixed models (GLMMs) were used to assess the potential species effect on absolute TRA, assuming a zero-inflated negative binomial distribution for oocysts counts. For each antibody type, treatment, antibody concentration and mosquito species were tested as fixed effects whilst replicate feeds were included as a random effect to correct for variance between feedings [37]. Estimates of TBA as the relative reduction in oocyst prevalence (using GLMMs with a binomial distribution) are presented in Additional file 1: Table S1, Additional file 2: Figure S1, Additional file 3: Figure S2 and Additional file 4: Figure S3. Intervention TBA has been shown to vary according to the level of parasitemia in the mosquito population (as assessed by mean oocyst intensity in control mosquitoes) [37, 38]. Since the overall number of oocysts might vary between mosquito species, TRA is used for the comparison between mosquito species in the main text. All statistics were carried out on the complete dataset though the figures and TRA estimates were obtained by using independent GLMMs for each individual antibody. To aid visual clarity, experiments where oocyst intensities were higher in test mosquitoes than controls were given an efficacy of zero in the figures but were left unaltered in the statistical analyses.

\section{Results}

The mean oocyst intensity and the prevalence of mosquitoes infected with oocysts in An. gambiae and $A n$. stephensi that were fed the same blood meal without any interventions were directly compared (Fig. 1a, b). Mean oocyst intensities in control feeds ranged from 2.9 to 113.7 in An. stephensi (oocyst prevalence 70-100\%) and 4.4 to 80.1 for $A n$. gambiae (oocyst prevalence $80-100 \%$ ). There was a broad correlation across the whole dataset between oocyst intensities in An. gambiae and An. stephensi (Spearman's correlation coefficient $r=0.92$, 95\% confidence interval (CI): 0.71-0.98, $P<0.0001)$. Absolute average numbers of oocysts were not significantly different between $A n$. gambiae and An. stephensi in control groups; 25.32 (95\% CI: 15.13-34.27) oocysts/mosquitoes for $A n$. stephensi vs 22.77 (95\% CI: 19.67-32.58) oocysts/mosquitoes for An. gambiae (GLM, $z_{(\mathrm{nb} \text {. of observations }=2194)}=1.13$, $P=0.18$. The absolute prevalence of oocysts in An. stephensi (83.79\%; 95\% CI: 59.27-83.81) was statistically significantly higher than in $A n$. gambiae $(73.26 \%$; 95\% CI: 64.33-93.84; $\left.z_{(2194)}=2.55, P=0.01\right)$.

The shape of the relationship between oocyst prevalence and oocyst intensity was tested in a larger dataset where the two mosquito species were not fed on the same blood sources. This comprises all SMFA experiments conducted between January 2011 and January 2013. The shape of the relationship between prevalence 

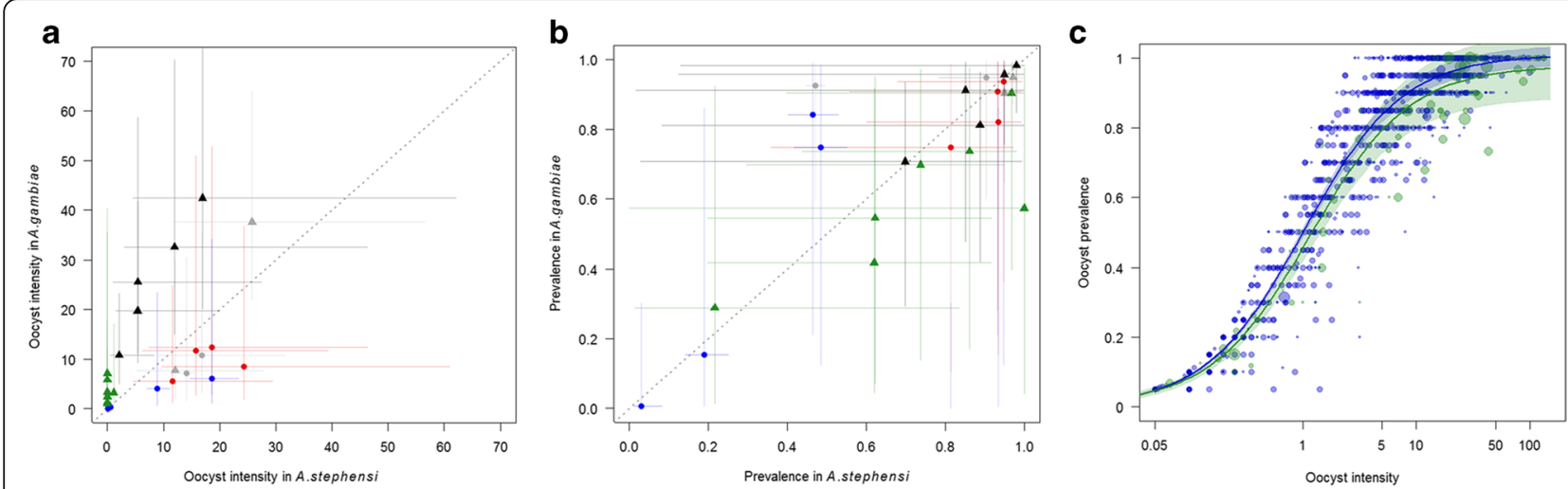

Fig. 1 Prevalence and oocyst intensity in An. stephensi and An. gambiae mosquitoes. a Mean oocysts intensity (dots) with 95\% confidence intervals (lines) in An. gambiae depending on mean oocyst intensity with 95\% confidence intervals for An. stephensi, for control groups (grey), mAb Pfs48/45-85RF45.1 (blue), mAb Pfs48/45-85RF45.5 (red), mAb Pfs25-32F81 (green) and mAb Pfs25-4B7 (black). b Mean prevalence in oocysts (dots) with 95\% confidence intervals (lines) in An. gambiae depending on mean prevalence with 95\% confidence intervals for An. stephensi. Same color codes as for panel a. c Relationship between oocysts prevalence and intensity for An. stephensi (blue) and An. gambiae (green). The line and shaded area represent the predicted relationship and predicted confidence interval. Each data point represents the mean oocyst intensity and oocyst prevalence in a single group of mosquitoes. The size of each data point represents the size of the mosquito group analyzed. Data were collected from both control and experimental feeds between 6 and 9 days post infection. In total 1635 separate feedings comprising 25,574 mosquito dissections are shown: 150 feeds with An. gambiae (green) comprising 2691 mosquitoes and 1485 feeds with An. stephensi (blue) comprising

22,883 mosquitoes

and intensity was similar for both species (Fig. 1c), suggesting the distribution of oocysts across mosquitoes is the same within An. gambiae and An. stephensi.

\section{Assessments of transmission-reducing activity of monoclonal antibodies}

TRA of four monoclonal antibodies, targeting Pfs48/45 (mAb 85RF45.1 and mAb 85RF45.5) and Pfs25 (mAb 32F81 and mAb 4B7) was investigated using An. stephensi and $A n$. gambiae. In experiments performed in both species, there was a very strong correlation between SMFA estimates using An. stephensi and An. gambiae (Spearman's correlation coefficient $r=0.80$, 95\% CI: 0.65-0.89\%, $\mathrm{P}<0.0001)$. If experiments were ranked in order of magnitude of TRA, the two species did not rank all experiments in the same order. Six mAb concentrations combinations resulted in $>80 \%$ TRA in An. stephensi (mAb 85RF45.1 at 10 and $2.5 \mu \mathrm{g} / \mathrm{ml} ; \mathrm{mAb} 32 \mathrm{~F} 81$ at 15, 10 and $5 \mu \mathrm{g} / \mathrm{ml} ; \mathrm{mAb} 4 \mathrm{~B} 7$ at $94 \mu \mathrm{g} / \mathrm{ml}) ; 4$ of these also showed > 80\% TRA in An. gambiae (mAb 85RF45.1 at 10 and $2.5 \mu \mathrm{g} / \mathrm{ml} ; \mathrm{mAb} 32 \mathrm{~F} 81$ at 15 and $10 \mu \mathrm{g} / \mathrm{ml}$ ) (Fig. 2). All highly active mAb concentrations tested in our laboratory colony of $A n$. gambiae also showed high efficacy in An. stephensi. For anti-Pfs 25 antibody concentrations with intermediate TRA, the efficacy was sometimes slightly overestimated in An. stephensi. Similar patterns were seen between species for transmission-blocking activity (TBA), showing no significant difference for all mAbs (mAb 85RF45.1, $z_{(2133)}=0.67, P=0.50 ; \mathrm{mAb}$ 85RF45.5, $z_{(2969)}=-0.45, P=0.65 ; \mathrm{mAb} 4 \mathrm{~B} 7, z_{(1070)}=-0.93, P=0.35$; mAb 32F81, $z_{(2594)}=-1.66 P=0.096$ ) (Additional files 2 :
Figure S1, Additional file 3: Figure S2). However, variance in TBA estimates was greater than TRA estimates since TBA is heavily dependent on control oocysts intensity [37, 38]. Control oocyst intensities vary considerably between experiments and, although this does not affect the validity of TRA estimates, high control oocyst intensities

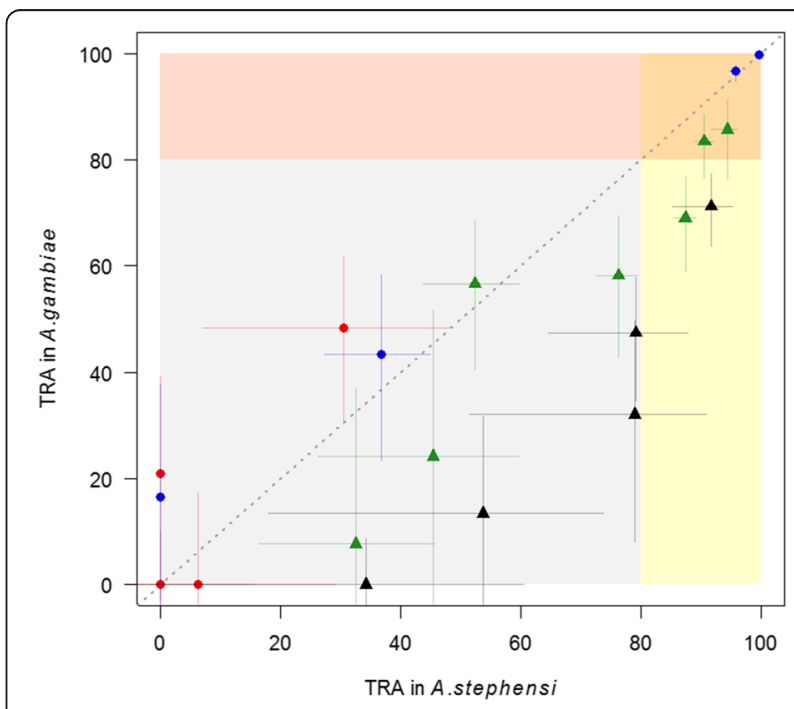

Fig. 2 The ranking of estimates of TRA of antibodies against Pfs $48 / 45$ (mAb 85RF45.1 and mAb 85RF45.5) and Pfs25 (mAb 32 F81 and mAb $4 \mathrm{B7}$ ) in An. gambiae and An. stephensi mosquitoes. TRA of transmission effective mAb 85RF45.1 (blue), 85RF45.5 (red), 32F81 (green) and 4B7 (black) in An. gambiae depending on TRA in An. stephensi mosquitoes. Dots and triangles represent the predicted TRA, while lines represent 95\% confidence intervals in An. gambiae and An. stephensi 
frequently lead to situations where oocyst prevalence is $100 \%$ and a considerable reduction in oocyst density is required before oocyst prevalence is affected, making TBA as outcome measure less relevant. For our experimental approach, TRA estimates were therefore considered more robust and more relevant than TBA estimates.

Although TRA outcomes of different mAb concentrations were ranked in broadly the same order, the actual TRA estimates showed some differences between $A n$. gambiae and An. stephensi, demonstrated by a significant interaction term between intervention and mosquito species using GLMM analysis $\left(z_{(8766)}=-2.71\right.$, $P=0.0067)$. The difference between mosquito species varied between antibody type and the efficacy of the intervention. To illustrate this, the impact of varying titers on TRA was assessed separately for the different antibodies.

The highly potent mAb 85RF45.1 showed high levels of TRA in both mosquito species. At a concentration of $10 \mu \mathrm{g} / \mathrm{ml}$, the number of oocysts was reduced by $99.8 \%$ (95\% CI: 99.5-99.9\%) and 99.9\% (95\% CI: 99.56-99.98\%) in An. stephensi and An. gambiae, respectively (Fig. 3a). At a concentration of $2.5 \mu \mathrm{g} / \mathrm{ml}$ near complete blockade was also observed for both species [An. stephensi: $96.2 \%$ (95\% CI: 94.3-97.4); An. gambiae: 96\% (95\% CI: 93.597.5)] while more variable levels of TRA were observed at lower concentrations. For mAb 85RF45.1, there was no significant effect of mosquito species on TRA and on the association between $\mathrm{mAb}$ concentration and TRA $z_{(2133)}=-0.45, P=0.65$ and $z_{(2133)}=-1.66, P=0.10$, respectively).

$\mathrm{mAb} 85 \mathrm{RF} 45.5$ is a much less potent $\mathrm{mAb}$ against Pfs48/45 [29] and at none of the tested concentrations was TRA consistently above the arbitrary threshold of $80 \%$ for reproducible SMFA experiments [39]. TRA estimates for 85RF45.5 were similar between An. stephensi and An. gambiae mosquitoes (Fig. 3b) and there was no significant effect of mosquito species on TRA and on the association between mAb concentration and TRA $z_{(2969)}=0.87, P=0.38$ and $z_{(2969)}=-0.59, P=0.55$, respectively).

For mAb 32F81 against Pfs25 we found high TRA activity in both mosquitoes species at concentrations of $15 \mu \mathrm{g} / \mathrm{ml}$ [An. stephensi: 91.2\% (95\% CI: 87.3-93.8) TRA; An. gambiae: $82.8 \%$ (95\% CI: 66.6-91.1)] and $10 \mu \mathrm{g} / \mathrm{ml}[$ [An. stephensi: 89.6\% (95\% CI: 80.1-94.4); An. gambiae: $82.5 \%$ (95\% CI: 6.0-96.8) TRA]. Below $10 \mu \mathrm{g} / \mathrm{ml}$, TRA activity remained relatively high in An. stephensi [ $5 \mu \mathrm{g} / \mathrm{ml}: 85.8 \%$ (95\% CI: 74.0-92.2); $2.5 \mu \mathrm{g} / \mathrm{ml}: 76.3 \%$ (95\% CI: 50.4-88.7)] but dropped below statistically significant levels in An. gambiae (Fig. 3c). The same pattern was observed for antibody mAb 4B7 against Pfs25: we found high TRA in both mosquitoes species at concentrations of $94 \mu \mathrm{g} / \mathrm{ml}[A n$. stephensi: 92\% (95\% CI: 86.4-95.3), An. gambiae: 67\% (95\%
CI: 52.8-77.0)]. Below this concentration, TRA remained relatively high in An. stephensi [23.5 $\mu \mathrm{g} / \mathrm{ml}: 78.4 \%$ (95\% CI: 67.7-85.6); $5.9 \mu \mathrm{g} / \mathrm{ml}: 49.6 \%$ (95\% CI: 25.3-66.0)] but dropped in An. gambiae [23.5 $\mu \mathrm{g} / \mathrm{ml}: 44.7 \%$ (95\% CI: 16.1-63.6\%); $5.9 \mu \mathrm{g} / \mathrm{ml}$ : below significant levels] (Fig. 3d). Anopheles stephensi supported a significantly higher TRA than An. gambiae for both anti-Pfs 25 $\mathrm{mAb}\left(32 \mathrm{~F} 81, z_{(2594)}=-3.95, \quad P<0.0001 ; 4 \mathrm{~B} 7\right.$, $\left.z_{(1070)}=-4.68, P<0.0001\right)$. This difference was significantly reduced by increasing antibody concentration in 4B7 $\left(z_{(1070)}=-4.21, P<0.0001\right)$.

Independent experiments were conducted at the Laboratory of Malaria and Vector Research (LMVR) using a very similar SMFA protocol [17] and provide data that support the above findings. In those experiments three mouse monoclonal antibodies (3E12, anti-Pfs $48 / 45$ $\mathrm{mAb}$; 1B3, anti-Pfs $230 \mathrm{mAb}$; 4B7, anti-Pfs $25 \mathrm{mAb}$ ) were tested by SMFA; no difference was observed in TRA between the two mosquito species for anti-Pfs48/45 and anti-Pfs230 antibodies whilst TRA estimates were higher in An. stephensi for anti-Pfs $25 \mathrm{mAb}$ (Additional file 5: Figure S4).

\section{Assessments of transmission-reducing activity of human serum $\lg G$}

Twenty-six human serum samples were selected and tested in direct paired experiments with the same parasite material and feeders from the same feeder chain used for An. stephensi and An. gambiae. Four of 5 samples from expatriates showed $\geq 99 \%$ TRA; the other sample showing negligible activity in the SMFA. Serum from one gametocyte carrier from Cameroon and 4 from Burkina Faso showed high and reproducible levels of TRA (84-100\%). All other field samples showed intermediate to low levels of TRA (Additional file 6: Table S2). There was a very strong association between SMFA estimates using An. stephensi and An. gambiae (Spearman's correlation coefficient $r=0.85,95 \%$ CI: 0.71-0.93, $P<0.0001$; Fig. 4).

Overall, TRA was significantly higher in An. stephensi than in An. gambiae mosquitoes (as expressed by the interaction term between species and intervention, GLMM $P<0.01)$ but with an average difference of only $12.8 \%(95 \%$ CI:16.2-19.9\%). As was seen in the previous analyses the greatest difference was observed at lower TRA estimates, with sera giving $>80 \%$ TRA being highly comparable in this experiment (Fig. 4). TBA estimates also showed a good correlation, and no significant difference, between An. stephensi and An. gambiae (Additional file 4: Figure S3).

\section{Discussion}

We compared TRA estimates of antibodies obtained in the SMFA using the commonly used An. stephensi and the important African malaria vector An. gambiae. There 


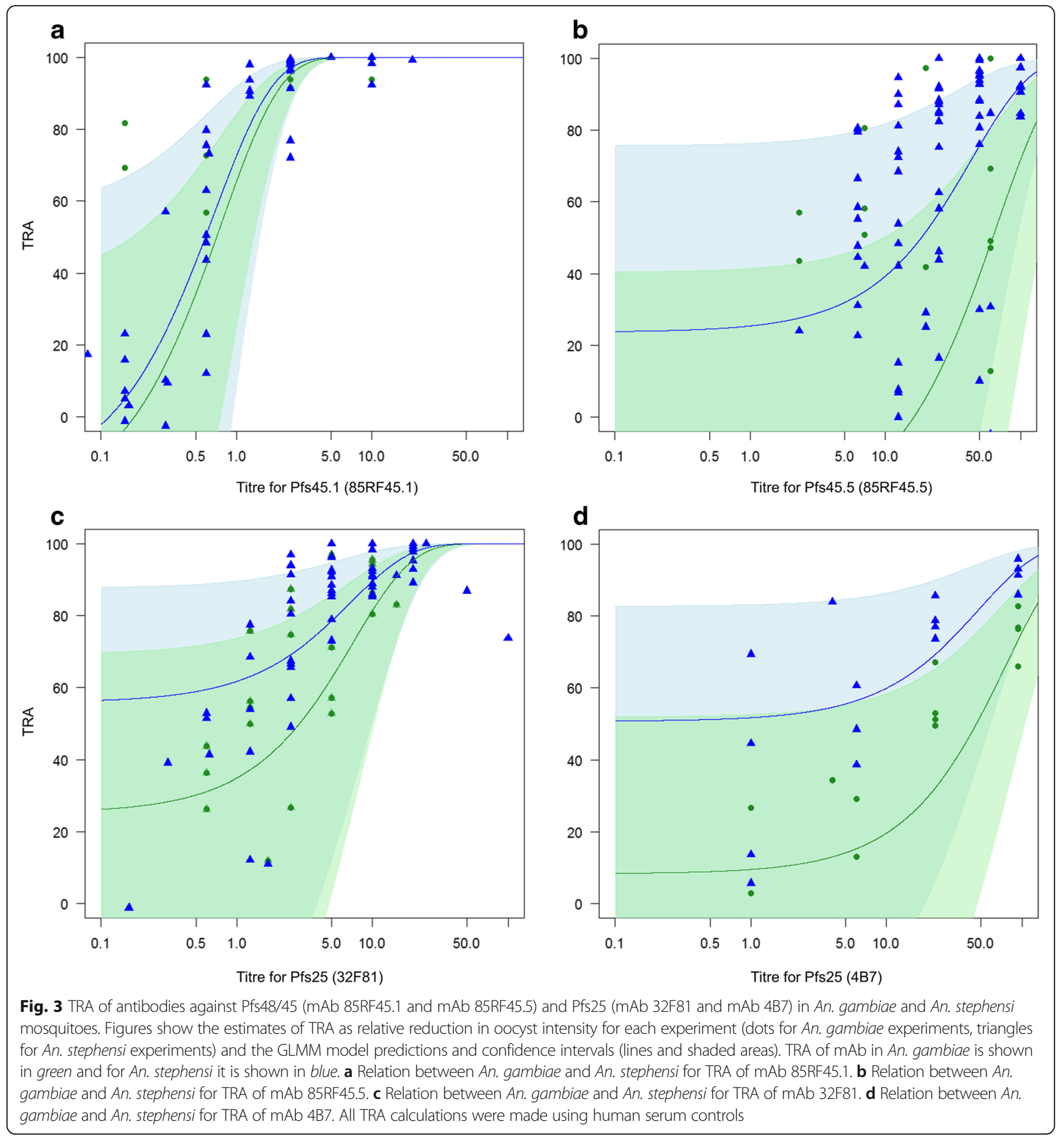

was a strong correlation in TRA efficacy estimates generated using the two mosquito species. For pre-fertilization anti-Pfs $48 / 45$ monoclonal antibodies and for the highest concentrations of post-fertilization anti-Pfs 25 antibodies tested here, we found no differences in absolute TRA estimates between the mosquito species. When partially effective concentrations of antibodies against Pfs 25 were used, we observed higher TRA estimates for An. stephensi.
Anopheles stephensi is commonly used as mosquito vector in the SMFA as well-established colonies are highly permissive and allow high oocyst and sporozoite densities to be achieved [17, 28]. Several characteristics of An. stephensi make this species markedly different from vectors that play a prominent role in the natural transmission of P. falciparum in Africa. An. gambiae has a potent thioester-containing protein-1 (TEP1) dependent immune 


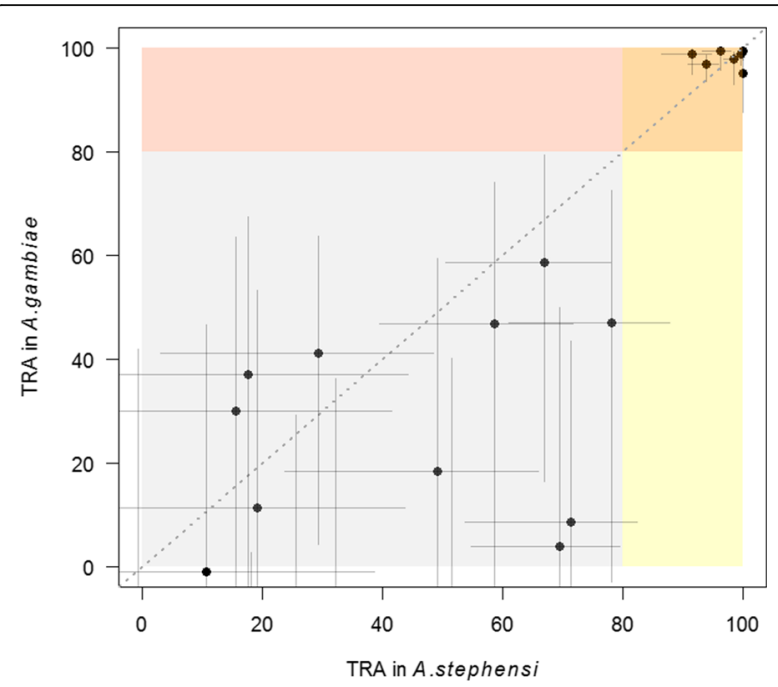

Fig. 4 TRA of human serum lgG from Cameroon and Burkina Faso and serum lgG from Dutch expatriate donor SP in An. gambiae and An. stephensi mosquitoes. All TRA calculations were made using FCS controls. TRA calculated using mean oocyst intensity assessments from oocyst intensity data from feeds with human serum IgG. R was calculated based on deviation from a perfect linear association $(x=y)$

system that is effective against Plasmodium infection. In our An. gambiae colony silencing TEP1 results in markedly higher infection rates with several $P$. falciparum strains [40]. The TEP1 response is absent or at least markedly lower in An. stephensi $[24,41]$. The difference in the interaction between Plasmodium and different anophelines is illustrated by the Plasmodium Pfs 47 gene that is essential to avoid TEP-1 mediated killing in An. gambiae but plays no evident role in sporogonic development in An. stephensi $[18,41]$. In addition, expression profiles of proteolytic enzymes during digestion of the blood meal vary between $A n$. stephensi and An. gambiae mosquitoes $[42,43]$, which may affect parasite survival and infectivity. The composition of bacterial midgut flora can also vary between mosquito species and may further influence the efficiency of Plasmodium transmission [44-48] although this may be less important in our mosquito colonies that were both maintained in the same laboratory.

Despite these differences, our findings provide no evidence for considerable differences between An. stephensi and An. gambiae in assessing TRA for pre-fertilization antibodies, reflected by indistinguishable TRA estimates for mAb against Pfs48/45 and highly similar TRA estimates for IgG from sera of naturally exposed individuals where antibodies are exclusively expected against prefertilization antigens [9]. Similarly, the highest concentrations of $\mathrm{mAb}$ against the post-fertilization antigen Pfs25 used in our experiments, effectively reduce transmission in both An. stephensi and An. gambiae. Our findings are less conclusive for lower, partially effective, concentrations of two different sources of anti-Pfs 25 mAb where TRA estimates appeared significantly higher for An. stephensi. Pfs 25 is translationally repressed in gametocytes and expressed in zygote and ookinete stages where it facilitates midgut invasion $[49,50]$. A previous study reported that Pfs25 specific IgG antibodies from immunized mice had comparable TRA efficacy in $A n$. gambiae and An. stephensi mosquitoes based on median number of oocysts but, similar to our findings, the lowest IgG concentration resulted in higher infection rates in An. gambiae. [51]. Whilst independent experiments in a different laboratory confirmed statistically significant higher estimate of TRA in An. stephensi compared to An. gambiae for the post-fertilization antibody Pfs 25 4B7 (Additional file 5: Figure S4), further work should elucidate whether this difference between mosquito species is also apparent for other post-fertilization antibodies and whether this reflects differences in antibody effectiveness that may need to be sustained for $>20 \mathrm{~h}$ in the mosquito gut to prevent further sporogonic development after ookinetes have formed [13, 52].

We designed our experiments to test TRA using reductions in oocyst density as the primary outcome measure. Ultimately, oocyst prevalence or the proportion of infected mosquitoes is a more relevant outcome to predict the community impact of interventions [5], an impact that may only become apparent after multiple transmission cycles [53]. Reductions in oocyst intensity (TRA) are typically less dependent on the efficiency of transmission and are strongly associated with reductions in oocyst prevalence (TBA) [37]. Our approach thus describes a suitable first step in candidate prioritization where multiple concentrations of transmission-blocking compounds are tested repeatedly using informative oocyst exposure in a highly permissive mosquito vector. However, compounds with activities downstream of gametocyte development might require testing in different species combinations to confirm potency in a range of epidemiologically relevant mosquito species. Our findings suggest that the SMFA with An. stephensi is an appropriate system for an important initial screen for TRA of transmission-blocking compounds. Ultimately, lead candidates will require testing at a range of oocyst intensities [5, 37] and ideally against multiple parasite strains. Testing candidates in feeding experiments with lower parasite exposure than routinely used in the SMFA (i.e. lower oocyst prevalence and intensity in control mosquitoes) will be of particular relevance for partially effective interventions that may still have a considerable impact on transmission [53]. Since the SMFA will always fall short of the natural situation of malaria transmission, testing interventions in the direct membrane feeding assays (DMFA) using gametocyte carriers with naturally circulating gametocyte isolates at natural densities and local mosquito species may provide additional information [54] that will help bridge the gap between in 
vitro candidate prioritization and predicting the efficacy of candidate interventions in real life.

\section{Conclusions}

Our study shows that monoclonal antibodies of target vaccines have comparable levels of transmission reduction in Anopheles stephensi and Anopheles gambiae mosquitoes. In addition, human serum IgG sample also have comparable levels of transmission reduction in An. stephensi and An. gambiae mosquitoes. These finding support the use of An. stephensi in the SMFA for target prioritization. However, compounds with activities downstream of gametocyte development may require additional testing in different species combinations to confirm potency in a range of epidemiologically relevant mosquito species.

\section{Additional files}

Additional file 1: Table S1. TRA and TBA estimates of human serum IgG samples. lgG concentration is shown in $\mathrm{mg} / \mathrm{ml}$. TRA estimates are shown as \% reduction in oocyst intensity with $95 \% \mathrm{Cl}$ and TBA estimates are shown as \% reduction in oocyst prevalence with 95\% Cl. (XLSX $31 \mathrm{~kb}$ )

Additional file 2: Figure S1. The ranking of estimates of TBA of antibodies against Pfs48/45 (mAb 85RF45.1 and mAb 85RF45.5) and Pfs 25 (mAb 32F81 and $\mathrm{mAb} 4 \mathrm{BB}$ ) in An gambiae and An. stephensi mosquitoes. TBA of transmission effective mAb 85RF45.1 (blue), mAb 85RF45.5 (red), mAb 32F81 (green) and mAb 4B7 (black) in An. gambiae depending on TBA in An. stephensi mosquitoes. Dots and triangles represent the predicted TBA, while lines represent 95\% confidence intervals in An. gambiae and An. stephensi. (TIFF $1302 \mathrm{~kb}$ )

Additional file 3: Figure S2. TBA of antibodies against Pfs48/45 ( $\mathrm{mAb}$ 85RF45.1 and mAb 85RF45.5) and Pfs25 (mAb $32 F 81$ and mAb 4B7) in An gambiae and An. stephensi mosquitoes. Figures show the estimates of TBA as relative reduction in oocyst prevalence for each experiment (dots for An. gambiae experiments, triangles for An. stephensi experiments) and the GLMM model predictions and confidence intervals (lines and shaded areas). TBA of $\mathrm{mAb}$ in An. gambiae is shown in green and for An. stephensi it is shown in blue. A Relation between An. gambiae and An. stephensi for TBA of mAb 85RF45.1 B Relation between An. gambiae and An. stephensi for TBA of mAb 85RF45.5 C Relation between An. gambiae and An. stephensi for TBA of mAb 32F81. D Relation between An. gambiae and An. stephensi for TBA of mAb 4B7. All TRA calculations were made using human serum controls. (TIFF $4772 \mathrm{~kb}$ )

Additional file 4: Figure S3. Correlation between ranking of TBA of human lgG samples in An. stephensi and An. gambiae. TBA of human serum IgG in An. gambiae depending on TBA in An. stephensi mosquitoes. Dots represent the predicted TBA, while lines represent 95\% confidence intervals in An. gambiae and An. stephensi. (TIFF $1082 \mathrm{~kb}$ )

Additional file 5: Figure S4. Outcomes of independently conducted experiments with mouse monoclonal antibodies against Pfs $48 / 45$ (mAb 3E12), Pfs230 (mAb 1B3) and Pfs 25 (mAb 4B7). Presented are estimates of transmission reducing activity in An. stephensi (blue) and An. gambiae (red). Box plots indicate median TRA with quartiles and range. The $n$ indicates the number of experiments. $P$-values are for the comparisons between mosquito species. (TIFF $1206 \mathrm{~kb}$ )

Additional file 6: Table S2. Overview of excluded SMFA experiments. Controls in experiments where prevalence was $<70 \%$ are highlighted in red. (TIFF $262 \mathrm{~kb}$ )

\section{Abbreviations}

Cl: Confidence interval; FCS: Foetal calf sera; GLMMS: Generalized linear mixed models; LMVR: Laboratory of Malaria and Vector Research; mAb: Monoclonal antibody; SMFA: Standard membrane feeding assay; SSM-
VIMT: Sexual-sporogonic-mosquito vaccines that interrupt malaria transmission; TBA: Transmission-blocking activity; TEP1: Thioester-containing protein-1; TRA: Transmission-reducing activity; VIMT: Vaccines that interrupt malaria transmission

\section{Acknowledgements}

The authors are grateful to Jolanda Klaassen, Astrid Pouwelsen, Laura PelserPosthumus and Jacqueline Kuhnen for their work on mosquito breeding and handling of the infected mosquitoes.

\section{Funding}

This work was supported by the PATH Malaria Vaccine Initiative and funds from the European Union's Seventh Framework Program (FP7/2007-2013), the latter under grant agreements $N^{\circ} 242,095$ (EVIMalar) N ${ }^{\circ} 223,736$ (TRANSMALARIABLOC) and N²23,601 (MALVECBLOK). TB is further supported by a VIDI fellowship from the Netherlands Organization for Scientific Research (NWO; project number 016.158.306). KM and CAL are supported in part by the intramural program of the National Institute of Allergy and Infectious Disease/NIH.

Availability of data and materials

The datasets used and/or analysed during the current study are available from the corresponding author on reasonable request.

\section{Authors' contributions}

$M E, K M, I M, A C, C L, M M, R S$ and TB designed experiments. ME, KM, GJVG, SR, $M V-B, W G, R S-S$ and $D D$ performed experiments. ME, AB, KM, WS, IM, AC, PB, $S R, R S, T C$ and TB analysed the data. ME, AB, WS, RS, TC and TB wrote the manuscript. All authors read and approved the final manuscript.

Ethics approval and consent to participate

Ethical approval was obtained from the Centre Muraz Institutional Ethics Committee under agreement number 0003-2009/CE-CM. The protocol conforms to the declaration of Helsinki on ethical principles for medical research involving human subjects (version 2002) and informed written consent were obtained from all volunteers.

Consent for publication

Not applicable.

\section{Competing interests}

The authors declare that they have no competing interests.

\section{Publisher's Note}

Springer Nature remains neutral with regard to jurisdictional claims in published maps and institutional affiliations.

\section{Author details}

'Department of Medical Microbiology, Radboud University Medical Center, Nijmegen, The Netherlands. ${ }^{2}$ MRC Centre for Outbreak Analysis and Modelling, Department of Infectious Disease Epidemiology, Imperial College London, London, UK. ${ }^{3}$ National Institute of Allergy and Infectious Diseases, Laboratory of Malaria and Vector Research, National Institutes of Health, Rockville, MD, USA. ${ }^{4}$ Institut de Recherche pour le Développement, UMR MIVEGEC UM-CNRS 5290-IRD 224, Montpellier, France. Institut de Recherche en Sciences de la Santé, Bobo Dioulasso, Burkina Faso. ${ }^{6}$ Institute of Biodiversity, Animal Health and Comparative Medicine, College of Medical, Veterinary and Life Sciences, University of Glasgow, Glasgow, UK. 'PATH Malaria Vaccine Initiative, Washington DC, USA. ${ }^{8}$ Department of Immunology and Infection, London School of Hygiene and Tropical Medicine, London, UK.

Received: 6 March 2017 Accepted: 1 October 2017

Published online: 17 October 2017

\section{References}

1. Bhatt S, Weiss DJ, Cameron E, Bisanzio D, Mappin B, Dalrymple U, et al. The effect of malaria control on Plasmodium falciparum in Africa between 2000 and 2015. Nature. 2015:526(7572):207-11.

2. Griffin JT, Hollingsworth TD, Okell LC, Churcher TS, White M, Hinsley W, et al. Reducing Plasmodium falciparum malaria transmission in Africa: a modelbased evaluation of intervention strategies. PLoS Med. 2010;7(8):20711482. 
3. Alonso PL, Brown G, Arevalo-Herrera M, Binka F, Chitnis C, Collins F, et al. A research agenda to underpin malaria eradication. PLoS Med. 2011;8(1): e1000406.

4. The malERA Consultative Group on Vaccines. A research agenda for malaria eradication: vaccines. PLoS Med. 2011;8(1):e1000398.

5. Sauerwein RW, Bousema T. Transmission blocking malaria vaccines: Assays and candidates in clinical development. Vaccine. 2015;33(52):7476-82.

6. Nikolaeva D, Draper SJ, Biswas S. Toward the development of effective transmission-blocking vaccines for malaria. Expert Rev Vaccines. 2015;14(5): 653-80.

7. Wu Y, Sinden RE, Churcher TS, Tsuboi T, Yusibov V. Development of malaria transmission-blocking vaccines: from concept to product. Adv Parasitol. 2015:89:109-52.

8. Vaughan JA, Noden BH, Beier JC. Population dynamics of Plasmodium falciparum sporogony in laboratory-infected Anopheles gambiae. J Parasitol. 1992;78(4):716-24.

9. Stone WJ, Dantzler KW, Nilsson SK, Drakeley CJ, Marti M, Bousema T, Rijpma SR. Naturally acquired immunity to sexual stage $P$. falciparum parasites. Parasitology. 2016;143(2):187-98.

10. van Dijk MR, Janse CJ, Thompson J, Waters AP, Braks JA, Dodemont HJ, et al. A central role for P48/45 in malaria parasite male gamete fertility. Cell. 2001;104(1):153-64.

11. Quakyi IA, Carter R, Rener J, Kumar N, Good MF, Miller LH. The 230-kDa gamete surface protein of Plasmodium falciparum is also a target for transmission-blocking antibodies. J Immunol. 1987;139(12):4213-7.

12. Rener J, Graves PM, Carter R, Williams JL, Burkot TR. Target antigens of transmission-blocking immunity on gametes of Plasmodium falciparum. J Exp Med. 1983;158(3):976-81

13. Vermeulen AN, Ponnudurai T, Beckers PJ, Verhave JP, Smits MA, Meuwissen $\mathrm{JH}$. Sequential expression of antigens on sexual stages of Plasmodium falciparum accessible to transmission-blocking antibodies in the mosquito. J Exp Med. 1985;162(5):1460-76.

14. Kaslow DC, Quakyi IA, Syin C, Raum MG, Keister DB, Coligan JE, et al. A vaccine candidate from the sexual stage of human malaria that contains EGF-like domains. Nature. 1988;333(6168):74-6.

15. Armistead JS, Morlais I, Mathias DK, Jardim JG, Joy J, Fridman A, et al. Antibodies to a single, conserved epitope in Anopheles APN1 inhibit universal transmission of Plasmodium falciparum and Plasmodium vivax malaria. Infect Immun. 2014;82(2):818-29.

16. Mathias DK, Jardim JG, Parish LA, Armistead JS, Trinh HV, Kumpitak C, et al. Differential roles of an anopheline midgut GPI-anchored protein in mediating Plasmodium falciparum and Plasmodium vivax ookinete invasion. Inf Genet Evol. 2014;28:635-47.

17. Miura K, Deng B, Tullo G, Diouf A, Moretz SE, Locke E, et al. Qualification of standard membrane-feeding assay with Plasmodium falciparum malaria and potential improvements for future assays. PLoS One. 2013;8(3):e57909.

18. Stone WJ, Churcher TS, Graumans W, van Gemert GJ, Vos MW, Lanke KH, et al. A scalable assessment of Plasmodium falciparum transmission in the standard membrane feeding assay using transgenic parasites expressing GFP-luciferase. J Infect Dis. 2014;210(9):1456-63.

19. Sinka ME, Bangs MJ, Manguin S, Rubio-Palis Y, Chareonviriyaphap T, Coetzee M, et al. A global map of dominant malaria vectors. Parasit Vectors. 2012;5:69.

20. Kamali M, Marek PE, Peery A, Antonio-Nkondjio C, Ndo C, Tu Z, et al. Multigene phylogenetics reveals temporal diversification of major African malaria vectors. PLoS One. 2014:9(4):e93580.

21. Collins WE, McClure H, Strobert E, Skinner JC, Richardson BB, Roberts JM, et al. Experimental infection of Anopheles gambiae s.s., Anopheles freeborni and Anopheles stephensi with Plasmodium malariae and Plasmodium brasilianum. J Am Mosq Control Assoc. 1993;9(1):68-71.

22. Vaughan JA, Hensley L, Beier JC. Sporogonic development of Plasmodium yoelii in five anopheline species. J Parasitol. 1994;80(5):674-81.

23. Hume JC, Tunnicliff M, Ranford-Cartwright LC, Day KP. Susceptibility of Anopheles gambiae and Anopheles stephensi to tropical isolates of Plasmodium falciparum. Malar J. 2007:6:139.

24. Jaramillo-Gutierrez G, Rodrigues J, Ndikuyeze G, Povelones M, MolinaCruz A, Barillas-Mury C. Mosquito immune responses and compatibility between Plasmodium parasites and anopheline mosquitoes. BMC Microbiol. 2009:9:154

25. Feldmann AM, Ponnudurai T. Selection of Anopheles stephensi for refractoriness and susceptibility to Plasmodium falciparum. Med Vet Entomol. 1989;3(1):41-52
26. Meister S, Agianian B, Turlure F, Relogio A, Morlais I, Kafatos FC, Christophides GK. Anopheles gambiae PGRPLC-mediated defense against bacteria modulates infections with malaria parasites. PLoS Pathog. 2009;5(8): e1000542.

27. Ponnudurai $T$, Lensen $A H$, Leeuwenberg AD, Meuwissen JH. Cultivation of fertile Plasmodium falciparum gametocytes in semi-automated systems. 1. Static cultures. T Roy Soc Trop Med H. 1982;76(6):812-8.

28. Ponnudurai T, Lensen AH, Van Gemert GJ, Bensink MP, Bolmer M, Meuwissen JH. Infectivity of cultured Plasmodium falciparum gametocytes to mosquitoes. Parasitology. 1989;98(Pt 2):165-73.

29. Roeffen W, Teelen K, van As J, vd Vegte-Bolmer M, Eling W, Sauerwein R. Plasmodium falciparum: production and characterization of rat monoclonal antibodies specific for the sexual-stage Pfs48/45 antigen. Exp Parasitol. 2001; 97(1):45-9.

30. Mulder B, Roeffen W, Sauerwein R, Tchuinkam T, Boudin C, Verhave JP. AntiPfs 25 monoclonal antibody 32F81 blocks transmission from Plasmodium falciparum gametocyte carriers in Cameroon. T Roy Soc Trop Med H. 1996; 90(2):195.

31. Barr PJ, Green KM, Gibson HL, Bathurst IC, Quakyi IA, Kaslow DC Recombinant Pfs 25 protein of Plasmodium falciparum elicits malaria transmission-blocking immunity in experimental animals. J Exp Med. 1991;174(5):1203-8

32. van der Kolk M, De Vlas SJ, Saul A, van de Vegte-Bolmer M, Eling WM, Sauerwein RW. Evaluation of the standard membrane feeding assay (SMFA) for the determination of malaria transmission-reducing activity using empirical data. Parasitology. 2005;130(Pt 1):13-22.

33. Harris C, Morlais I, Churcher TS, Awono-Ambene P, Gouagna LC, Dabire RK, et al. Plasmodium falciparum produce lower infection intensities in local versus foreign Anopheles gambiae populations. PLoS One. 2012;7(1):e30849.

34. Outchkourov NS, Roeffen W, Kaan A, Jansen J, Luty A, Schuiffel D, et al. Correctly folded Pfs48/45 protein of Plasmodium falciparum elicits malaria transmission-blocking immunity in mice. Proc Natl Acad Sci USA. 2008;105: 4301-5.

35. R Development Core Team R. A language and environment for statistical computing, vol. 1; 2006.

36. Fournier DA, Skaug HJ, Ancheta J, lanelli J, Magnusson A. Maunder MN, et al. AD Model Builder: using automatic differentiation for statistical inference of highly parameterized complex nonlinear models. Opti. Meth Softw. 2012; 27(2):233-49.

37. Churcher TS, Blagborough AM, Delves M, Ramakrishnan C, Kapulu MC Williams AR, et al. Measuring the blockade of malaria transmission - an analysis of the standard membrane-feeding assay. Int J Parasitol. 2012; 42(11):1037-44

38. Miura K, Swihart BJ, Deng B, Zhou L, Pham TP, Diouf A, et al. Transmissionblocking activity is determined by transmission-reducing activity and number of control oocysts in Plasmodium falciparum standard membranefeeding assay. Vaccine. 2016;34(35):4145-51.

39. Miura K, Stone WJ, Koolen KM, Deng B, Zhou L, van Gemert GJ, et al. An inter-laboratory comparison of standard membrane-feeding assays for evaluation of malaria transmission-blocking vaccines. Malaria J. 2016;15:463.

40. Eldering M, Morlais I, van Gemert GJ. van de Vegte-Bolmer M, Graumans W, Siebelink-Stoter R, et al. Variation in susceptibility of African Plasmodium falciparum malaria parasites to TEP1 mediated killing in Anopheles gambiae mosquitoes. Sci Rep. 2016;6:20440.

41. Molina-Cruz A, Garver LS, Alabaster A, Bangiolo L, Haile A, Winikor J, et al. The human malaria parasite Pfs47 gene mediates evasion of the mosquito immune system. Science. 2013;340(6135):984-7.

42. Chen XG, Mathur $G$, James AA. Gene expression studies in mosquitoes. Adv Ggenet. 2008;64:19-50.

43. Nolan T, Petris E, Muller HM, Cronin A, Catteruccia F, Crisanti A. Analysis of two novel midgut-specific promoters driving transgene expression in Anopheles stephensi mosquitoes. PLoS One. 2011;6(2):e16471.

44. Pumpuni CB, Demaio J, Kent M, Davis JR, Beier JC. Bacterial population dynamics in three anopheline species: the impact on Plasmodium sporogonic development. Am J Trop Med Hyg. 1996;54(2):214-8.

45. Meister S, Kanzok SM, Zheng XL, Luna C, Li TR, Hoa NT, et al. Immune signaling pathways regulating bacterial and malaria parasite infection of the mosquito Anopheles gambiae. Proc Natl Acad Sci USA. 2005;102(32):11420-5.

46. Dong $Y$, Manfredini F, Dimopoulos $G$. Implication of the mosquito midgut microbiota in the defense against malaria parasites. PLoS Pathog. 2009:5(5): e1000423. 
47. Noden BH, Vaughan JA, Pumpuni CB, Beier JC. Mosquito ingestion of antibodies against mosquito midgut microbiota improves conversion of ookinetes to oocysts for Plasmodium falciparum, but not $P$. yoelii. Parasitol Int. 2011;60(4):440-6.

48. Tchioffo MT, Boissiere A, Churcher TS, Abate L, Gimonneau G, Nsango SE, et al. Modulation of malaria infection in Anopheles gambiae mosquitoes exposed to natural midgut bacteria. PLoS One. 2013;8(12):e81663.

49. Vlachou D, Lycett G, Siden-Kiamos I, Blass C, Sinden RE, Louis C. Anopheles gambiae laminin interacts with the P25 surface protein of Plasmodium berghei ookinetes. Mol Biochem Parasitol. 2001;112(2):229-37.

50. Tomas AM, Margos G, Dimopoulos G, van Lin LH, de Koning-Ward TF, Sinha R, et al. P25 and P28 proteins of the malaria ookinete surface have multiple and partially redundant functions. EMBO J. 2001;20(15):3975-83.

51. Kumar R, Angov E, Kumar N. Potent malaria transmission-blocking antibody responses elicited by Plasmodium falciparum Pfs25 expressed in Escherichia coli after successful protein refolding. Infect Immun. 2014;82(4):1453-9.

52. Dinglasan RR, Kalume DE, Kanzok SM, Ghosh AK, Muratova O, Pandey A, Jacobs-Lorena M. Disruption of Plasmodium falciparum development by antibodies against a conserved mosquito midgut antigen. Proc Natnl Acad Sci USA. 2007;104(33):13461-6.

53. Blagborough AM, Churcher TS, Upton LM, Ghani AC, Gething PW, Sinden RE. Transmission-blocking interventions eliminate malaria from laboratory populations. Nat Commun. 2013;4:1812.

54. Bousema T, Churcher TS, Morlais I, Dinglasan RR. Can field-based mosquito feeding assays be used for evaluating transmission-blocking interventions? Trends Parasitol. 2013;29(2):53-9.

\section{Submit your next manuscript to BioMed Central and we will help you at every step:}

- We accept pre-submission inquiries

- Our selector tool helps you to find the most relevant journal

- We provide round the clock customer support

- Convenient online submission

- Thorough peer review

- Inclusion in PubMed and all major indexing services

- Maximum visibility for your research

Submit your manuscript at www.biomedcentral.com/submit 